Results $30 \%$ of YP consented to take part. Data are available for the first 94 participants (mean [SD] age: 15.16 [1.24] years; $96 \%$ female). Those with learning disability and young people of black ethnicity were over-represented compared to the local population (18\% vs. $2.5 \%$, and $23 \%$ vs. $11 \%$, respectively).

$15 \%$ had a history of previous non-consensual sex and $44 \%$ were known to social services. 39\% had sought help for mental health difficulties in the previous year and $37 \%$ had previously self-harmed.

Participants experienced vaginal rape in $72 \%$ of cases, oral rape in $40 \%$ and anal rape in $13 \%$. 44\% of assaults involved physical violence and 9\% involved a weapon. $29 \%$ involved alcohol and 14\% involved drugs. 36\% were stranger assaults, $47 \%$ involved an acquaintance and the remainder involved partners or ex-partners (3\%), or a relative $(5 \%)$. Assailants were most commonly aged $15-20(41 \%)$ or $21-30$ (22\%), with $12 \%$ $<15$ years.

Early psychological outcomes: $73 \%$ had significant depressive symptoms, $90 \%$ had a high likelihood of post-traumatic stress disorder, 69\% met criteria for panic disorder or significant somatic symptoms, and $60 \%$ met criteria for generalised anxiety disorder.

Conclusions Adolescents accessing SARCs are a vulnerable population and exhibit high levels of psychological morbidity within six weeks of sexual assault. Longitudinal research is critically important for evaluating outcomes over time and to inform interventions and preventive programmes.

\section{P04 1 RCT OF A MOTIVATIONAL LIFESTYLE INTERVENTION (THE HEALTHY EATING AND LIFESTYLE PROGRAMME (HELP)) FOR OBESE YOUNG PEOPLE}

${ }^{1} \mathrm{D}$ Christie, ${ }^{2} \mathrm{~L}$ Hudson, ${ }^{2} \mathrm{~S}$ Costa, ${ }^{2} \mathrm{~A}$ Mathiot, ${ }^{1} \mathrm{R}$ Holt, ${ }^{3} \mathrm{~S}$ Kinra, ${ }^{4} \mathrm{~A}$ Kessel, ${ }^{5} \mathrm{CK}$ Wong, ${ }^{2}$ TJ Cole, ${ }^{6} \mathrm{~S}$ Morris, I Nazareth, ${ }^{2} \mathrm{RM}$ Viner. ${ }^{1}$ University College London Hospitals NHS Foundation Trust, London, UK; ${ }^{2}$ University College London Institute of Child Health, London, UK; ${ }^{3}$ London School of Tropical Medicine and Hygiene, London, UK; ${ }^{4}$ Public Health England, London, UK; ${ }^{5}$ Department of Pharmacology and Pharmacy, University of Hong Kong, Honk Kong; ${ }^{6}$ Department of Applied Health Research, University College London, London, UK; ${ }^{7}$ Department of Primary Care, University College London, London, UK

\subsection{6/archdischild-2015-308599.4}

Aims To assess whether a motivational multi-component lifestyle intervention delivered in the community was effective in reducing body mass index (BMI) and improving related health outcomes in obese adolescents.

Methods 174 obese adolescents (13-17 years old; 109 females) from a UK community setting were randomised into intervention or control arms. Intervention participants received 12 sessions across 6 months, addressing lifestyle behaviours and focusing on motivation to change and self-esteem rather than weight change. The intervention was delivered by trained Graduate Health Workers in community settings. Control participants received a single $2 \mathrm{~h}$ nurse-delivered session providing didactic weight management advice. The primary outcome was BMI change at 6 months. Secondary outcomes included body fat (impedance;), dieting behaviours, self-esteem and quality of life. Randomeffects linear regression was used to detect differences in endpoint outcomes between Intervention and control groups, adjusting for sex, age, and outcome value at the beginning of the intervention. The primary analyses used the intention to treat sample.
Results 145 (83.3\%) adolescents completed the intervention. Mean BMI across the whole group was $32.3 \mathrm{~kg} / \mathrm{m}^{2}$ (SD 4.4) at start and $32.6 \mathrm{~kg} / \mathrm{m}^{2}$ (SD 4.7) at the end of the intervention. We found no significant difference in the primary outcome (BMI) at 6 months: effect estimate -0.06 (95\% CI: -0.57 to 0.45 ) $\mathrm{p}=0.8)$. No significant differences were observed for changes in secondary outcomes (all p $>0.4$ ) between intervention and control groups at 6 months. Fidelity monitoring showed moderately strong fidelity to treatment. The process evaluation found that participants and their families found the intervention highly engaging, respectful and helpful in making behavioural changes.

Discussion We did not find evidence that a motivational multicomponent lifestyle modification intervention delivered in the community was effective in reducing BMI or improving health and well-being in a community sample of obese adolescents, despite moderately strong fidelity and process evidence that young people used the intervention to make changes in their lifestyle. Our findings suggest that obesity interventions with a strong theoretical basis and evidence of effectiveness when delivered by trained psychologists may not be effective when delivered at lower intensity in the community by entry-level health workers.

\section{P05 LONG-TERM EFFECTS OF ACUTE MALNUTRITION ON GROWTH AND BODY COMPOSITION IN MALAWIAN CHILDREN}

${ }^{1,2} \mathrm{~N}$ Lelijveld, ${ }^{1} \mathrm{~A}$ Seal, ${ }^{3} \mathrm{~J}$ Wells, ${ }^{2} \mathrm{R}$ Heyderman, ${ }^{2,4} \mathrm{M}$ Nyirenda, ${ }^{2,4} \mathrm{M}$ Kerac. ${ }^{1}$ Institute for Global Health, University College London, London, UK; ${ }^{2}$ Malawi-Liverpool Wellcome Trust, Blantyre, Malawi; ${ }^{3}$ London School of Hygiene and Tropical Medicine, London, UK; ${ }^{4}$ Institute of Child Health, University College London, London, UK

\subsection{6/archdischild-2015-308599.5}

Aims Severe Acute malnutrition (SAM) is an important cause of child mortality worldwide and most treatment to date has focused on reducing those deaths. However, with emerging evidence that early nutritional adversity affects adult health, it is vital that treatment strategies also start looking beyond short term outcomes at programme discharge. To do this, improved evidence on the long term implications is needed; in this study, we examined growth and body composition 7 years after an episode of SAM.

Methods We present latest data from a follow-up of 462 exmalnourished Malawian children, comparing their growth and body composition to both siblings and age/sex matched community controls. These are the known survivors of an original cohort of 1024 children admitted to a large Malawian nutrition ward, from 2006 to 2007, for treatment of SAM. The current round of follow-up is 7 years after the original episode of malnutrition. Linear regression is used to analyse interim anthropometric data.

Results To date, 321/412 (78\%) of searches have been successful. Median age of the ex-malnourished 'case' children was 9 yrs 2 months (range: 7-20 years). 79/321 (25\%) are HIV positive; $35 / 321(11 \%)$ died in the last six years. Cases are significantly more stunted and underweight than community controls. Waisthip ratio was significantly higher for cases suggestive of adverse body composition, however skinfold thickness ratio (subscapular +waist/tricep) was not significantly different between the groups. Sitting height ratio was also significantly higher for case children suggesting that torso length has been preserved and limb growth compromised. In addition, ex-malnourished case children had evidence of functional impairment with their hand-grip strength 
significantly weaker than that of community controls. Table 1 presents further details.

Conclusions These results indicate that SAM may be associated with a number of adverse long-term effects, including stunting, abnormal body composition and functional impairment. It will be crucial to identify effective strategies, not only to prevent SAM in the first place, but to improve long-term outcomes in SAM survivors. Interventions might include more proactive case finding to encourage earlier detection and continued follow-up after the initial treatment to support high risk children and families.

\begin{tabular}{|c|c|c|c|c|c|}
\hline \multicolumn{2}{|c|}{$\begin{array}{l}\text { Abstract P05 Table } 1 \\
\text { controls }\end{array}$} & \multicolumn{4}{|c|}{ Linear regression of cases vs community } \\
\hline Measurement & $\begin{array}{l}\text { Mean for } \\
\text { Case } \\
\text { (ex-SAM) } \\
\text { children }\end{array}$ & $\begin{array}{c}\text { Mean for } \\
\text { Community } \\
\text { control } \\
\text { children }\end{array}$ & $\begin{array}{c}\text { Cases vs } \\
\text { Community } \\
\text { Regression } \\
\text { Coefficient }\end{array}$ & $\begin{array}{c}95 \% \\
\text { Confid ence } \\
\text { Interv al }\end{array}$ & $\begin{array}{c}\text { p- } \\
\text { value }\end{array}$ \\
\hline Weight-for-age & $-1.6 z$-scores & $-1.2 z$-scores & -0.35 & $-0.61,-0.09$ & 0.01 \\
\hline Height-for-age & -1.8 z-scores & -1.3 z-scores & -0.48 & $-0.77,-0.18$ & $<0.001$ \\
\hline BMI-for-age & -0.9 z-scores & -0.7 z-scores & -0.22 & $-0.44,0.00$ & 0.06 \\
\hline $\begin{array}{l}\text { Waist:Hip ratio } \\
(\log )\end{array}$ & 0.91 & 0.88 & 0.03 & $0.01,0.06$ & 0.01 \\
\hline $\begin{array}{l}\text { Sitting } \\
\text { height/standing } \\
\text { height ratio }\end{array}$ & 52.2 & 51.8 & 0.55 & $0.16,0.94$ & 0.01 \\
\hline $\begin{array}{l}\text { Hang grip } \\
\text { strength (log) }\end{array}$ & $12.2 \mathrm{~kg}$ & $13.8 \mathrm{~kg}$ & -0.12 & $-0.18,-0.05$ & $<0.001$ \\
\hline $\begin{array}{l}\text { Skinfold } \\
\text { thickness r atio }\end{array}$ & 1.73 & 1.72 & -0.02 & $-0.12,0.07$ & 0.61 \\
\hline
\end{tabular}

\section{P06 DELIVERY OF A SCHOOL HEALTH AND NUTRITION PROGRAMME FOR KENYAN CHILDREN: A FEASIBILITY STUDY}

B Eder, J Bogie, D Magnus. Centre for Child and Adolescent Health, University of Bristol, Bristol, UK

\subsection{6/archdischild-2015-308599.6}

Aims Health is a key factor in enabling universal access to education. Children miss over 200 million days of school every year due to illness. Traditionally, vertical approaches have been used to individually tackle child health and nutrition problems such as malnutrition or malaria. However, these interventions overlook the complex interactions between them. We therefore sought to develop a comprehensive, integrated school health and nutrition programme to produce long-term and sustainable improvements to child health and education outcomes.

Methods A 3 year pilot study was carried out in a population of 2,000 children aged 5-16 in rural Western Kenya. The programme comprised school meals, vitamin A supplementation, insecticide-treated bed nets, deworming, hand washing and health education. Data were collected on programme delivery and on health, nutrition and education parameters.

Results 2103 children were dewormed using Albendazole, supplied with insecticide-treated bed nets and Vitamin A was given to all children under 5. School meals were provided daily to all children. Additional water points with soap were installed with a $32.5 \%$ observational increase in clean hands and $86 \%$ increased soap use. Preliminary results show increased school enrolment and reductions in school absenteeism (OR 0.70, 95\% CI $0.61-0.82, \mathrm{p}=<0.001$ ), in anaemia (OR $0.25,95 \%$ CI $0.16-0.40, \mathrm{p}=<0.001)$ and undernutrition, both stunting (OR 0.56, 95\% CI $0.48-0.65, \mathrm{p}=<0.001$ ) and underweight (OR $0.55,95 \%$ CI $0.42-0.73, \mathrm{p}=0.001$ ).

Conclusion Our experience shows that a comprehensive school health and nutrition programme is feasible and that monitoring for health and education outcomes is possible in this context. The data suggest improvements in childhood anaemia and nutrition in conjunction with improved school attendance and enrolment. Complex interventions for improving school health are feasible and can produce long-term benefits above and beyond vertical programme initiatives.

\section{P07 EVIDENCE-BASED GUIDANCE TO INFORM CONSENT SEEKING IN CHILDREN'S CRITICAL CARE TRIALS}

K Woolfall, L Frith, B Young. Institute of Psychology, Health and Society, University of Liverpool, Liverpool, UK

\subsection{6/archdischild-2015-308599.7}

Aims Challenges in seeking parents' consent for research at the point when their child is critically ill have been a significant barrier to improving treatments for children and conducting trials. In 2008 UK legislation was amended to enable consent for emergency research to be sought after a child has been given the investigational drug or device. This is known as deferred consent. CONNECT is the first UK study to explore parent and practitioner views and experiences of deferred consent in children's clinical care trials. It aimed to integrate evidence and ethical theory to inform practice guidelines to optimise recruitment and consent in this challenging setting.

Methods Mixed methods qualitative and quantitative study with 354 participants (292 parents, 39 nurses, 19 doctors and 4 Clinical Trials Unit practitioners). We integrated findings with previous research and ethical theory to develop draft guidance on deferred consent. The CONNECT advisory group and 32 key stakeholders (critical care practitioners, ethicists and parents) contributed to the development of the final CONNECT guidance.

Results Parents may be initially shocked or angered to discover their child can be entered into a trial without their prior consent. However practitioner explanations of why consent is deferred can help address parents' initial concerns and reassure them. Parents view deferred consent as more acceptable for trials of medical interventions already used in clinical practice than trials involving new interventions or a change in clinical practice. Practitioners experienced in deferred consent describe how families are receptive to the consent method, if conducted sensitively and if the timing is appropriate. CONNECT guidance provides recommendations to inform: 1) pre-trial research for potentially challenging trials; 2) publicising trials that use deferred consent; 3) seeking deferred consent; 4) seeking deferred consent when a child has died; 5) written trial information; and 6) child assent. Conclusion Those involved in the funding, design, conduct and ethical review of critical care trials can use CONNECT guidance to help ensure approaches to deferred consent are appropriate 